\title{
Design and Fabrication of a W-Band Linear Array of Planar Antennas and Study for the Enhancement of its Radiation Properties with a Chiral Metamaterial Cover
}

\author{
Jéssica Gutiérrez*, Óscar Fernández, Juan Pablo \\ Pascual, Álvaro Gómez, Antonio Tazón, Ángel Vegas \\ Department of Communications Engineering \\ University of Cantabria \\ Santander, Spain \\ \{jessica.gutierrez, oscar.fernandez, juanpablo.pascual, \\ alvaro.gomez, antonio.tazon, angel.vegas\}@unican.es
}

\author{
Naima Amar \\ Faculty of Sciences \\ Abdelmalek Essaadi University \\ Tetouan, Morocco \\ nai_amar@yahoo.fr
}

\begin{abstract}
This work presents the design, fabrication and measurements of a $\mathrm{W}$-band linear array of planar antennas. The structure of the antenna, which presents as benefit low cost and easy manufacture, is composed of three planar patches fed by slots. The simulation and the experimental results are compared showing a good agreement. In order to enhance the radiation properties, a chiral metamaterial cover is added to the antenna array. The chiral metamaterial cover is formed by two layers of mutually twisted planar metal rosettes in parallel planes and it is expected to enhance the directivity of the antenna array.
\end{abstract}

Keywords- W-band; antenna array; chiral metamaterial cover

\section{INTRODUCTION}

Taking advantage of interesting characteristics offered by Terahertz frequencies (high resolution, good penetration into nonconductor materials with low attenuation and not harmful for human beings health), numerous applications are being developed from investigations in this new field. Specially, we highlight imaging applications in $\mathrm{W}$ band, [1]-[2] in airport security: as remote detections of weapons, explosives or drugs, or even other applications in early detection of cancers, among others.

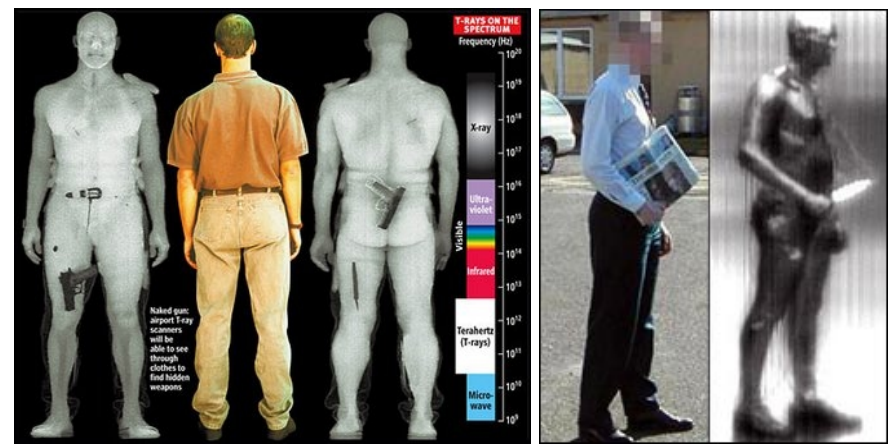

Figure 1. Imaging Applications. Detecting Concealed Weapons

This work has been partially supported by the MINECO of Spanish Government through the Research Projects CONSOLIDER CSD2008-00068 and CSD2008-00066, FEDER co-funded TEC2011-29126-C03-01 and TEC2010-21496-C03-01
Our main goal is to design and fabricate an antenna array to be included later as part of a radiometer system in the mentioned W-band (this frequency band seems to be the most technologically accessible). The structure of the antenna is based on a linear array of planar antennas excited by slots. Regarding to the performance, this type of antennas presents high directivity and gain, reasonable matching, good Side Lobe Level (SLL) and well backward radiation. Moreover, its easy manufacture and low cost are remarkable features.

Later, a chiral metamaterial cover is presented and added in the antenna layer structure in order to improve the radiation properties. With this novel cover, a higher gain and a narrower main lobe are expected to be obtained. The cover is composed by a circuit board with a pattern of rosettes printed on both metallization layers of the substrate as will be seen later [3].

\section{DESIGN OF THE ANTENNA ARRAY}

The complete design of our antenna array is composed by two parts: a waveguide-to-microstrip transition and the own linear patch antenna array excited by slots. This type of antenna is characterized by low cost and easy manufacturing.

The objective of this design is to obtain a good response of gain, with a value about $13 \mathrm{dBi}$ (typical gain in microstrip technology) and high directivity, initially centered around $96 \mathrm{GHz}$ and with a $-10 \mathrm{~dB}$ bandwidth about 7GHz. Different 3D electromagnetic simulators have been using during the antenna design [4],[5] to validate it.

\section{A. Waveguide-to-Microstrip Transition}

A waveguide-to-microstrip transition working in W-band is a necessary component to be included into the design of the antenna array. This is due to that, as it will be discussed later, WR10 waveguide is the standard for $\mathrm{W}$ band modules connectivity. Moreover, the PNA-X microwave Network Analyzer used during the experimental measurements needs

\footnotetext{
${ }^{1} 1 \mathrm{mils}=0.0254 \mathrm{~mm}$
} 
two auxiliary Millimeter-Wave VNA extension modules terminated in standard W-band waveguide accesses.

The waveguide-to-microstrip transition is designed using the Rogers ULTRALAM 2000 substrate $\left(\varepsilon_{\mathrm{r}}=2.5\right.$, $\operatorname{tg} \delta=$ 0.0019 , copper thickness $=18 \mu \mathrm{m}, \mathrm{h}=4 \mathrm{mils}$ ) and it is based on the previous design of a transition described in [6]. The design is shown in Fig. 2, where the different sections which constitute the microstrip line can be seen.

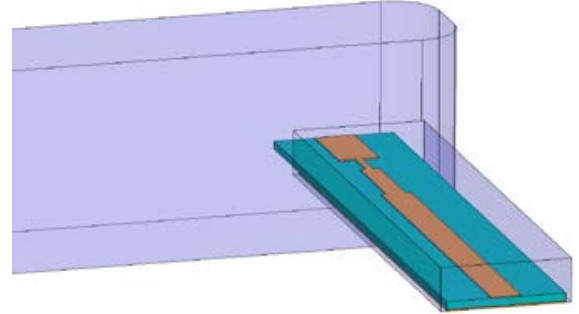

Figure 2. Waveguide-to-microstrip transition in W-band

In order to verify the correct behavior of the design, a backto-back transition (double waveguide-to-microstrip transition) has been fabricated and measured to characterize it. A comparison between simulation and measurements is shown in Fig. 3.

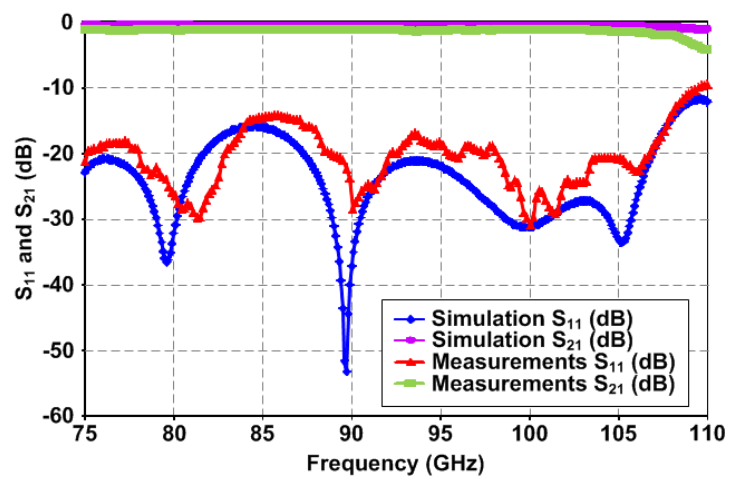

Figure 3. Simulation and experimental results of the back-to-back transition in W-band

\section{B. Complete Design of the Antenna Array}

The design of the linear antenna array composed of three patches fed by slots was initiated with a previous model and design of a single radiant patch.

Patch dimensions and slot dimensions were optimized taking into account input matching and gain. The patch dimensions are $1 \times 1.1 \mathrm{~mm}$ and the slot dimensions are $0.7 \times$ $0.09 \mathrm{~mm}$ and this patch is fed by $100 \Omega$-microstrip line.

The array was formed adding two more identical patches to

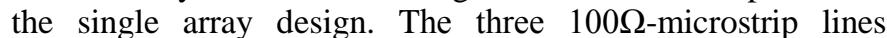
(dimensions: $3.7 \times 0.09 \mathrm{~mm}$ ) are connected by a $50 \Omega$ microstrip line of $0.26 \mathrm{~mm}$ length, which, at a distance of $0.5 \mathrm{~mm}$, is enclosed in a cut-off waveguide (dimensions: $1.14 \times$ $0.55 \mathrm{~mm}$ ) to propagate only the W-band Quasi-TEM microstrip mode. Other details in the design are: the phase center length between patches is $2.2 \mathrm{~mm}$ and the distance from the feed line to the case back is $0.5 \mathrm{~mm}$.
The characteristics of the different design layers with their metallization are the following:

- The low cost ULTRALAM 2000 substrate $(\varepsilon r=2.5$, $\operatorname{tg} \delta=0.0019, \mathrm{~h}=4$ mils and a copper thickness of 18 $\mu \mathrm{m})$ is used in the deeper layer in order to avoid the propagation of higher modes with assumable losses. This layer contains the $100 \Omega$ feed line located at its rear face and the ground plane where the slots are settled at the top face of the same layer.

- $\quad$ The intermediate layer is the radiant substrate, air, in this case, with a thickness of 5 mils. Two side supports composed of Rogers RT/Duroid 5880 substrate $(\mathrm{h}=$ 5mils) are necessary to hold this air gap.

- The top layer is fabricated with Rogers RT/Duroid 5880 substrate $(\varepsilon r=2.2$ and $\operatorname{tg} \delta=0.0009$ and $\mathrm{h}=$ 5mils). It acts as a radome and holds the metallic patches in its bottom face.

Finally, the waveguide-to-microstrip transition was connected to the linear array of planar antennas excited by slots and the complete antenna can be seen in Fig. 4 with other details added to become a more realistic structure, like metallic screws, to put together the different layers and the pieces of the metallic structure which were included in the final design.

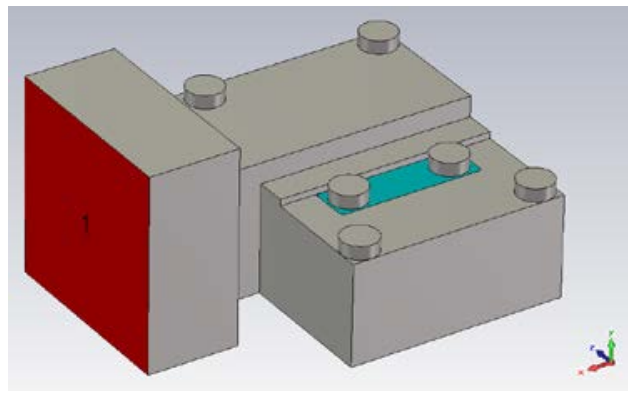

Figure 4. Complete structure of the antenna array

The input matching obtained from the 3D electromagnetic simulation is shown in Fig. 5. In this graph, we can see a -10dB bandwidth of about $7 \mathrm{GHz}$ with a minimum value at $96 \mathrm{GHz}$.

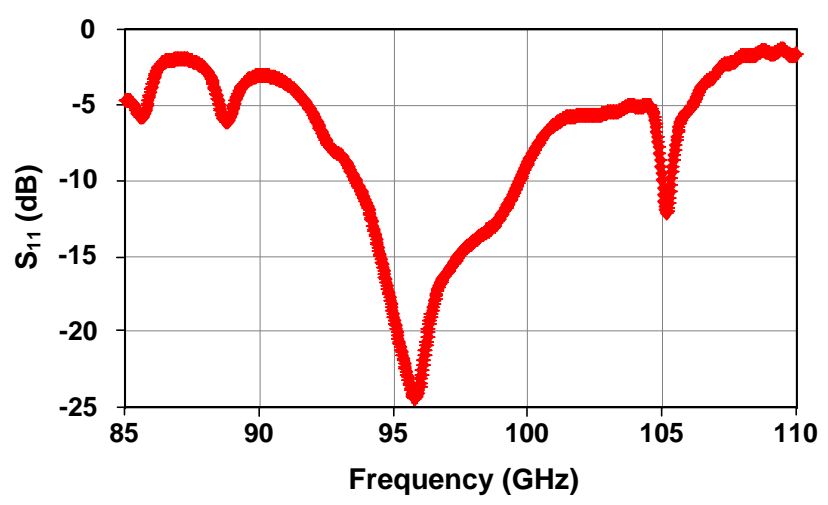

Figure 5. Input Matching $\left(\mathrm{S}_{11}\right)$ of the antenna array

The most important parameter in the design of the antenna is its gain, so it is calculated in the simulations and its results are plotted in Fig. 6. 


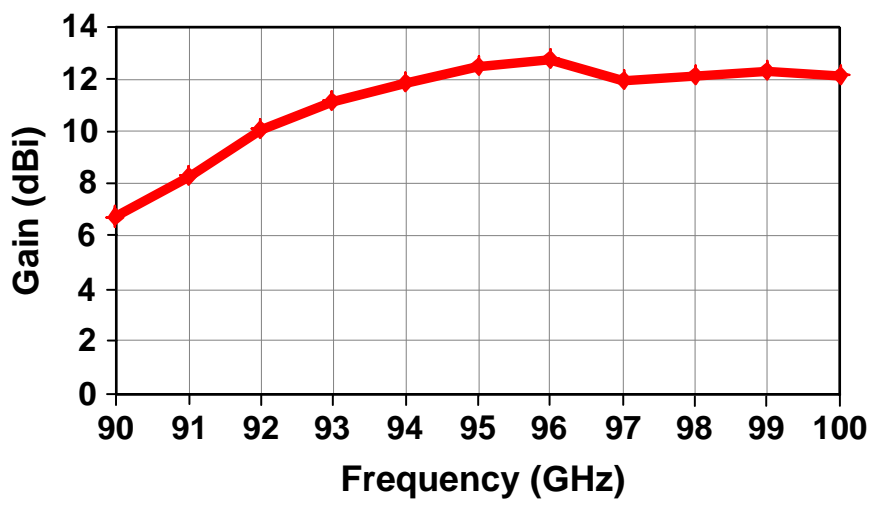

Figure 6. Gain of the antenna array

As it can be seen, a good gain value is obtained (around $13 \mathrm{dBi}$ ) according to the number of patch elements of the array.

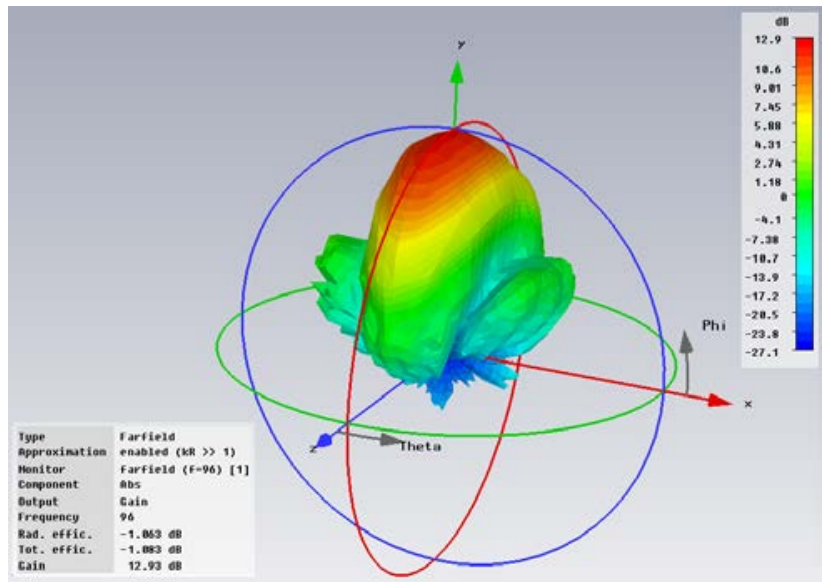

Figure 7. Radiation pattern of the antenna array

Finally, we show the 3D pattern radiation in far field at $96 \mathrm{GHz}$ (Fig. 7). A value of $13 \mathrm{dBi}$ is reached in this frequency and -14dB Side Lobe Level is obtained [7], which is a typical value in this type of structure. Moreover, the achievement of a negligible backward radiation value should be highlighted.

\section{Prototype Manufacturing}

The prototype of our antenna has been made up in two stages: the metal case (fabricated in two pieces) and the different layers of the radiant elements. The dimensions of the complete structure are $4 \times 3 \mathrm{~cm}$. The fabricated metal case is shown in Fig. 8.

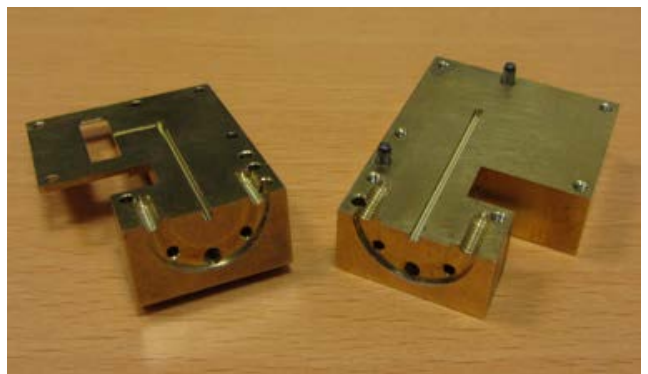

Figure 8. Photograhs of the metal case (two pieces) for the patch antenna array
The different layers that constitute the radiant elements are shown in Fig. 9, where the top and bottom faces of the lower layer and the top layer can be seen.

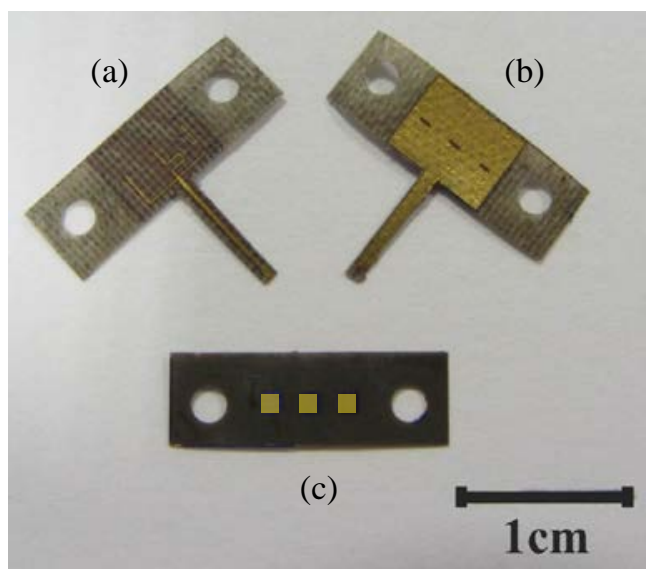

Figure 9. Photographs of the different layers of the complete radiant system. (a) feed lines and microstrip lines of the waveguide-to-microwave transition, (b) ground and slots and (b) patches of the array

Finally, all layers are placed within the metal case to complete the assembling of the prototype. A detail of the upper layer soldered on the metal case is displayed in Fig. 10.

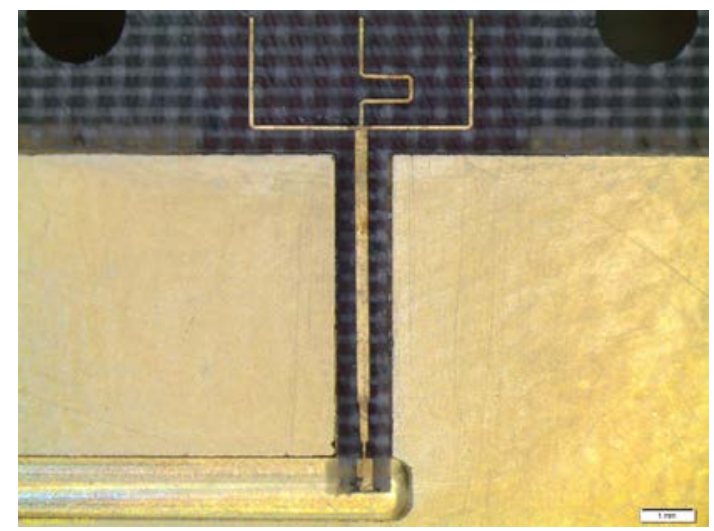

Figure 10. Zoom of the deeper layer within the complete radiant system

\section{EXPERIMENTAL MEASUREMENTS}

\section{A. Measurement Setup}

A N5242A PNA-X Microwave Network Analyzer was used for the experimental measurements. Two auxiliary Millimeter-Wave VNA extension modules were added to the input and output ports of the setup to expand the analyzer to millimeter frequencies, specifically to W-band (both heads present WR-10 waveguide access). The port 1 head is a transceiver/receiver module whereas the port 2 head is only a receiver module, therefore $S_{11}$ and $S_{21}$ parameters can be measured at the same time, but $\mathrm{S}_{22}$ and $\mathrm{S}_{12}$ parameters can be measured turning the DUT (Device Under Test) in a second measurement.

All measurements shown were done in far filed condition. The input matching was the first measurement done, placing the prototype antenna at the port 1 head. 
For the case of the realized gain, two steps were carried out. In first place, two identical horn antennas previously characterized were placed in the setup, measuring their $S_{21}$ parameter and calculating their gains (assumed equal) from this value. Then, one of these horn antennas was replaced by the antenna array under test, obtaining its realized gain, adding the differential value between the actual $S_{21}$ and the previous $S_{21}$ parameter, to the horn antenna gain.

Regarding to the radiation features, the radiation pattern in far field was obtained from two measurements. The elevation plane was carried out in first place, and the setup of the laboratory in this case can be seen in Fig. 11. So, the antenna array was placed in the port 1 and the horn antenna in the port 2. The scanning was made from $-65^{\circ}$ up to $+65^{\circ}$ in a 5 degrees step, moving the horn antenna respect to the array.

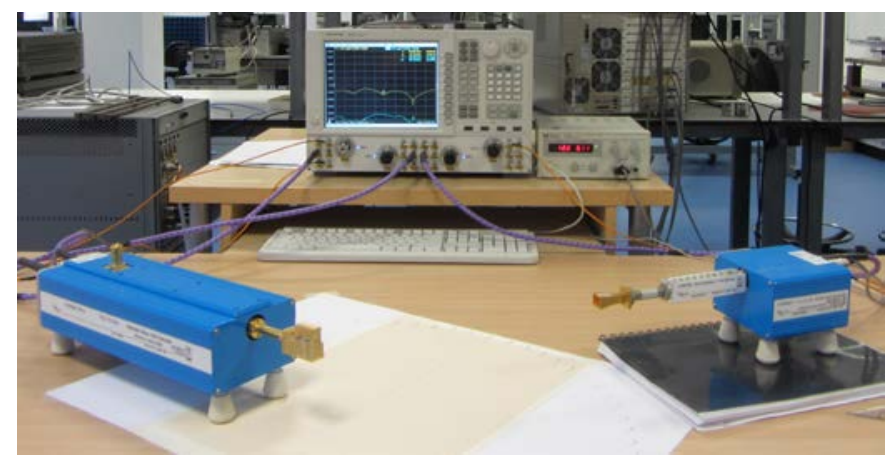

Figure 11. Setup for the 2D radiation pattern in elevation plane

To obtain the azimuth plane in a simple and easy way (see Fig. 12), the horn antenna was placed in this case in the port 1, and the antenna array in the port 2, turning its position in vertical sense respect to the measurements plane. Movement for obtaining the azimuth plane was done by the array, scanning from $-65^{\circ}$ up to $+65^{\circ}$ in a 5 degrees step.

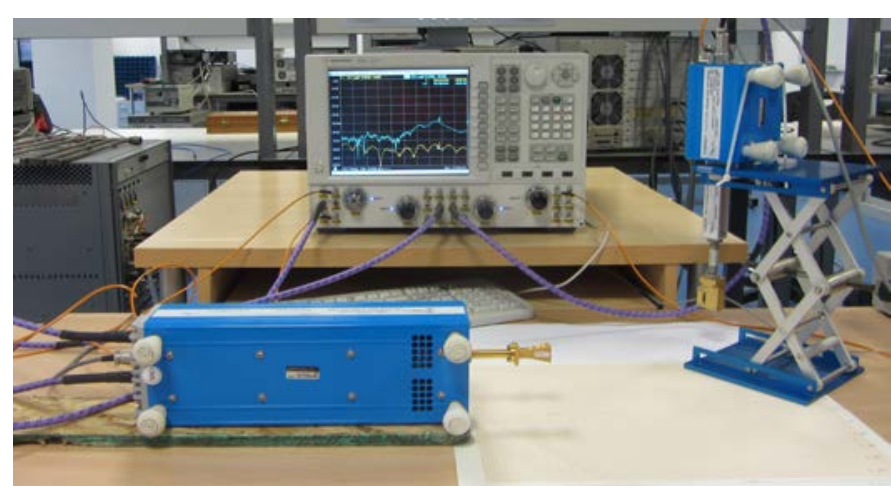

Figure 12. Setup for the 2D radiation pattern in azimuth plane

\section{B. Measurements Results}

In this section, all the results obtained from the experimental measurements are shown.

Measured input matching of the antenna array in far field conditions is compared in Fig. 13 with simulated results. From these results, we can see a good response with similar values although a frequency shift is observed, being the minimum $S_{11}$ simulated at $96 \mathrm{GHz}$ and the minimum $\mathrm{S}_{11}$ measured at 102.2GHz. In a second iteration, this shift could be corrected readjusting the design. This shift can be due to the gap between the top and deeper layer as well as the variations in the feed lines dimensions due to fabrication tolerance.

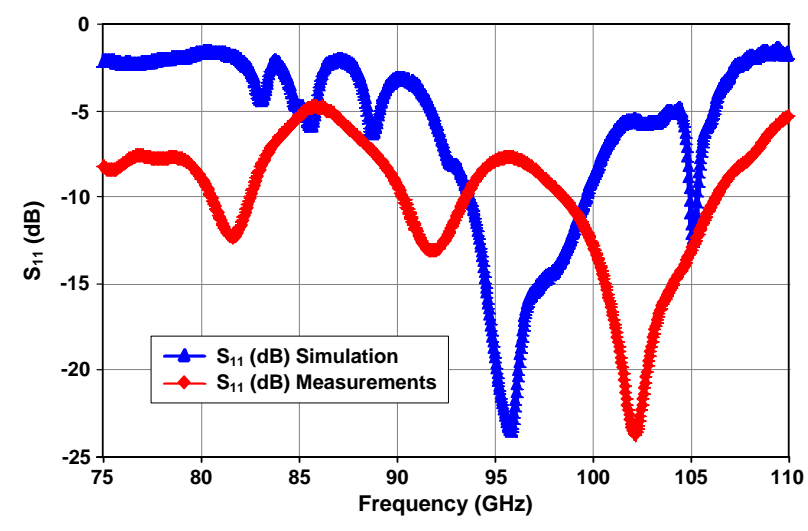

Figure 13. Comparison of the input matching between simulations and measurements

The Realized Gain was the second measurement carried out in the laboratory (see Fig. 14). The shape of the measured gain compared with the simulated gain is quite similar in the range of frequency, and the maximum value of the measured gain is $8.7 \mathrm{dBi}$, lower than the simulated value of $12.7 \mathrm{dBi}$. This difference can be due to additional losses of the transition, access, setup used, etc...

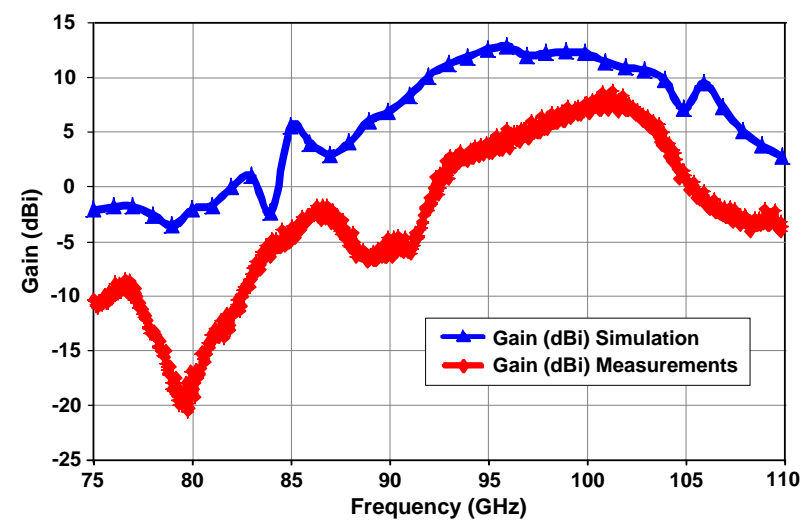

Figure 14. Gain: comparison between simulations and measurements

Finally, we present the 2D radiation pattern in elevation (Fig. 15) and azimuth (Fig. 16) planes. It is interesting to comment that these radiation patterns shown are represented at the minimum return loss frequency, which is $96 \mathrm{GHz}$ for the simulations and $102.2 \mathrm{GHz}$ for the measurements.

It can be observed in the comparison of both radiation patterns measured from $-65^{\circ}$ up to $+65^{\circ}$, but simulated in the whole range, an excellent result of the antenna array prototype with respect to the desired behavior. We can highlight the good value and shape of the side lobules regarding the main lobule in the elevation plane, as well as a lack of directivity in the other plane as it was expected. 


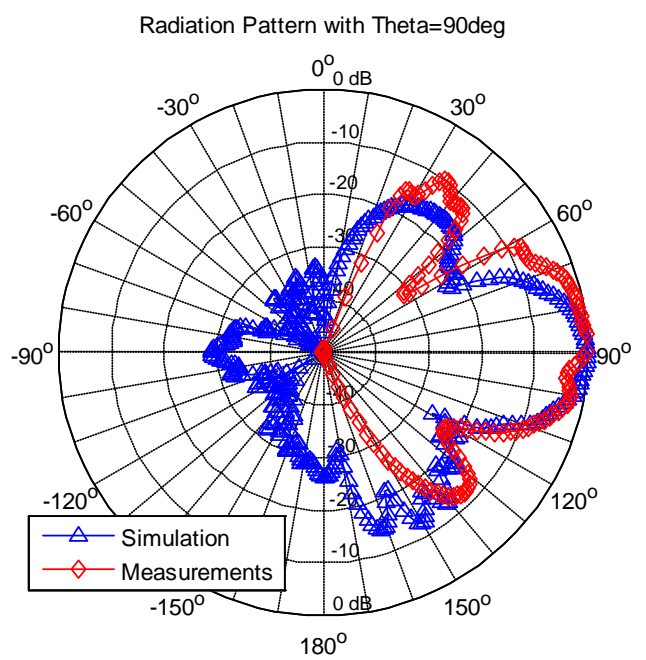

Figure 15. Radiation pattern of the elevation plane: simulations and measurements results

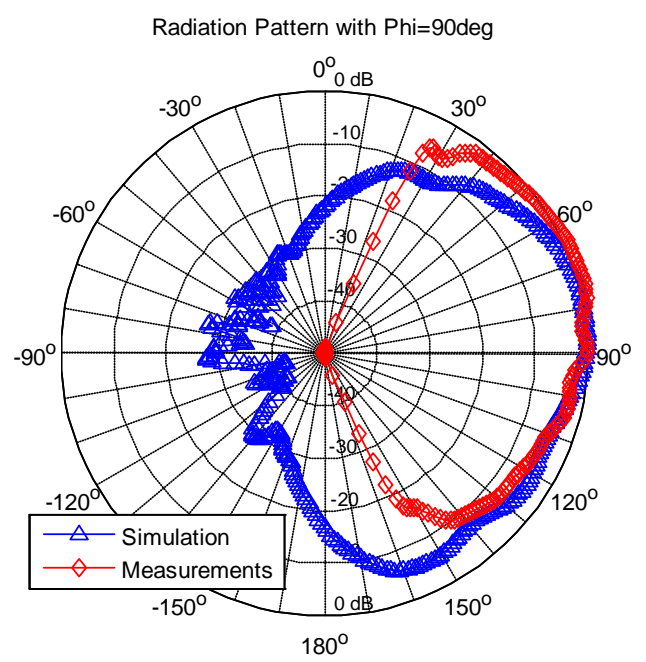

Figure 16. Radiation pattern of the azimuth plane: simulations and measurements results

Measured results of matching, gain and radiation pattern compare well with other similar antennas in the same band [8],[9].

\section{Chiral Metamaterial COVER to EnHANCE THE RADIATION PROPERTIES}

Good results have been obtained in the experimental measurements of the prototype antenna. As it was seen in Section IIIB, high directivity only exits in the elevation plane. Then, as a possible improvement of the directivity in the azimuth plane [10], a chiral metamaterial cover is presented in this section.

The chiral cover is characterized by a unit cell consisting of two planar metallic rosettes printed on both sides of a substrate (see Fig. 17), according to ref. [3]. The two rosettes are placed in parallel and one of them is twisted a certain angle respect to the other one. This condition provides the property of 3D chirality to this structure.

The dimensions of the unit cell are: radius $=166 \mu \mathrm{m}$, width $=80 \mu \mathrm{m}$ and a copper thickness of $18 \mu \mathrm{m}$. The relative twist of the rosettes is $15^{\circ}$, and the dimensions of the unit cell dielectric substrate are $1 \times 1 \mathrm{~mm}$, with a thickness of 6 mils. The relative constant is $2.2+\mathrm{j} \cdot 0.0009$.

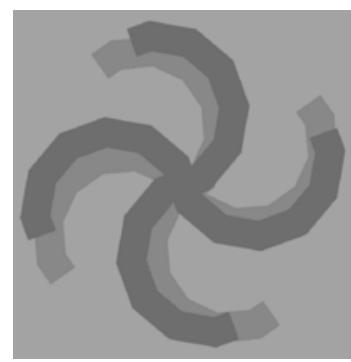

Figure 17. Chiral metamaterial cover unit cell

The final cover is composed by $7 \times 5$ aligned pairs of rosettes as can be seen in Fig. 18. Moreover, it is placed above the antenna array at a certain distance of it and between the two lateral screws, parallel and aligned to the radome.

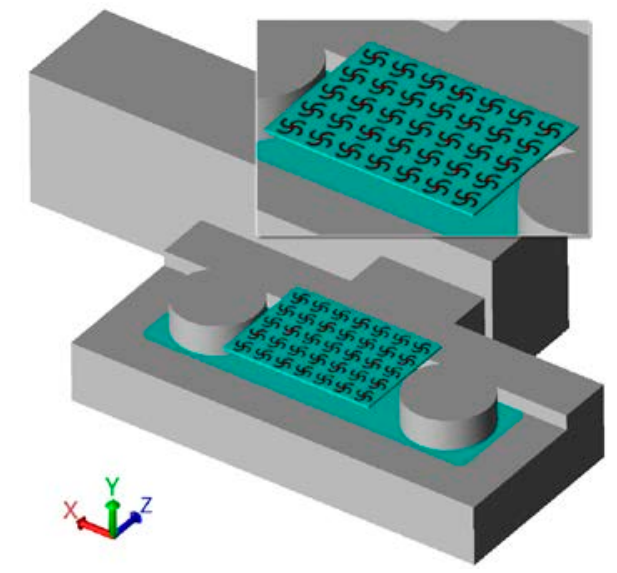

Figure 18. Antenna with chiral metamaterial cover. Inset: Zoom of the cover.

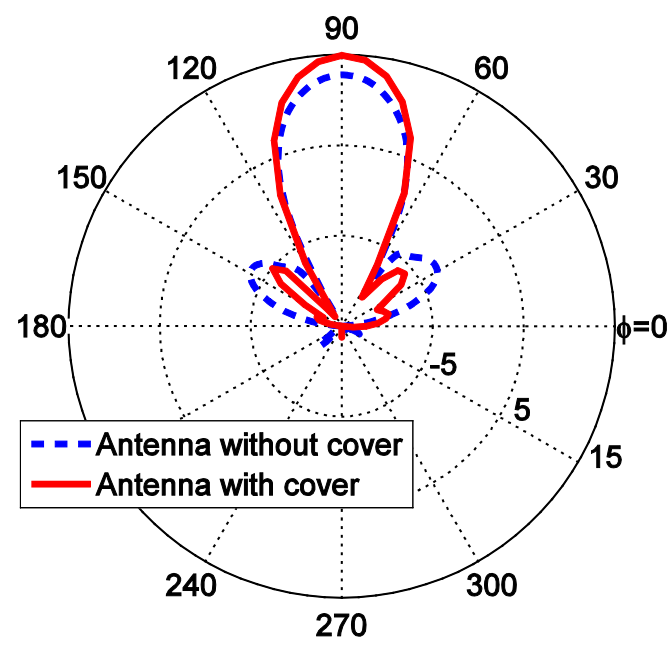

(a) 


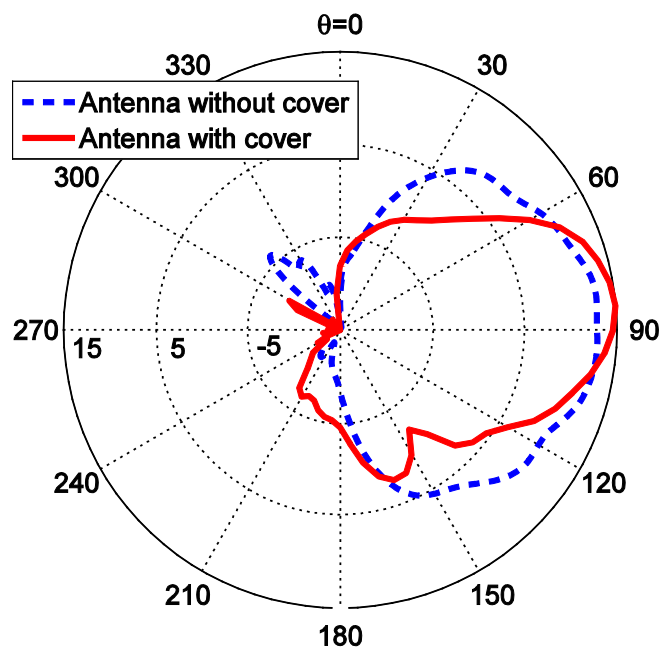

(b)

Figure 19. Radiattion pattern with and without cover (a) azimuth plane, (b) elevation plane

In Fig. 19, it is depicted a comparison of radiation pattern (azimuth and elevation plane) simulation results with and without the cover. On inspecting this figure, it can be seen how the simulated gain is improved $2 \mathrm{~dB}$ (from 13 to $15 \mathrm{dBi}$ ) when the cover is included in the antenna structure. Moreover, the Side Lobe Level decreases $4 \mathrm{~dB}$, from $-16 \mathrm{~dB}$ up to $-20 \mathrm{~dB}$.

Regarding to the radiation pattern main lobe, the cover produces a narrowing in the response. So, on one hand, in the elevation plane, the chiral metamaterial focuses the radiation pattern, as described in [11], in the broadside direction, reducing the $3 \mathrm{~dB}$ beam width $21^{\circ}$, decreasing it from $57^{\circ}$ to $36^{\circ}$. On the other hand, in the elevation plane, the main lobe $\mathrm{dB}$ beam width has not changed but the secondary lobes gain and beam width have been reduced.

Another aspect to remark in Fig. 19a, is that the main lobe in the elevation plane is deviated 5 degrees from broadside direction $(\theta=90, \phi=90)$. This deviation is due to a light misalignment between the three patches array and the cover.

\section{CONCLUSIONS}

In this paper, a design of a high directivity antenna array in $\mathrm{W}$-band is presented. This antenna is composed of three patches fed by slots and a waveguide-to-microstrip transition. This antenna has been fabricated and measured, and a comparison between simulation results and experimental measurements has been presented showing a good agreement in input matching, gain and radiation pattern.
Then, a chiral metamaterial cover has been added to the designed structure in order to evaluate the improvement in the antenna radiation features over all in the azimuth plane, where the antenna array does not have directivity. The cover has increased $2 \mathrm{~dB}$ the gain, reduced $21^{\circ}$ the $3 \mathrm{~dB}$ beam width and $4 \mathrm{~dB}$ in the Side Lobe Level.

\section{ACKNOWLEDGMENT}

Authors would like to thank to Sandra Pana for the fabricated samples of the available prototype and to Ángel Mediavilla for his help in tooling of the metal case.

\section{REFERENCES}

[1] S. Oka, H. Togo, N. Kukutsu and T. Nagatsuma, "Latest trends in millimeter-wave imaging technology”, Progress In Electromagnetics Research Letters, vol. 1, pp. 197-204, 2008.

[2] R. Appleby and H. B. Wallace, "Standoff detection of weapons and contraband in the $100 \mathrm{GHz}$ to $1 \mathrm{THz}$ region”, IEEE Trans. Antennas Propag., vol. 55, no. 11, pp.2944-2956, Nov. 2007.

[3] E. Plum, J. Zhou J. Dong, V. A. Fedotov, T. Koschny, C. M. Soukoulis and N. I. Zheludev, "Metamaterial with negative index due to chirality", Physical Review B, vol. 79, no. 3, pp. 035407-0355413, Jan. 2009.

[4] www.cst..com

[5] http://www.home.agilent.com/agilent/software.jspx?ckey=2216605\&id= $2216605 \&$ nid $=-11143.0 .00 \& l c=$ eng\&cc $=E S$

[6] J. Gutiérrez, K. Zeljami, J.P. Pascual, T. Fernández, A. Tazón, A. Mediavilla, E.S. Pana. "Yield-Oriented Design Protocol and Equivalent Circuit Model for W-Band E-Plane Waveguide-to-Microstrip Transitions". International Journal of RF and Microwave ComputerAided Engineering. DOI: 10.1002/mmce.20716. Volume 24, Issue 1, pp. 77-91. Jan. 2014

[7] N. Amar, L. Cabria, J. A. Garcia, A. Tazon, A. Mediavilla and F Marante, “A transmitting active array with spatially selective linealization," The Second European Conference on Antennas and Propagation, EuCAP 2007 , pp.1-5, 11-16, Nov. 2007

[8] S. Agarwal, N. P. Pathak, D. Singh. "Performance Comparison of Microstrip Patch Antenna for $94 \mathrm{GHz}$ Imaging Applications”. $7^{\text {th }}$ IEEE International Conference on Industrial and Information Systems (ICIIS), pp. 1-4. Aug. 2012

[9] A. Lamminen, J. Säily. "Wideband Stacked Patch Antenna Array on LTCC for W-Band”. Proceedings of the $5^{\text {th }}$ European Conference on Antennas and Propagation (EUCAP 2011), pp. 2962-2966. April 2011

[10] O. Fernández, A. Gómez, J. Gutiérrez, A. Tazón, A. Vegas, J.P. Pascual. "Enhancement of the radiation properties of a linear array of planar antennas with a chiral metamaterial cover" 9th Spanish Conference on Electron Devices (CDE), 2013, pp.223,226, 12-14 Feb. 2013. Valladolid, Spain. DOI: 10.1109/CDE.2013.6481383

[11] V.V. Gozhenko, J. Wigle, J. Vedral, B. Hendrix and A.O. Pinchuk, "Focusing effect of a metamaterial slab on the radiation pattern produced by a patch antenna”, Metamaterials, 2012. http://dx.doi.org/10.1016/j.metmat.2012.06.00 